\title{
IdeAs
}

Idées d'Amériques

$11 \mid 2018$

Modernités dans les Amériques : des avant-gardes à aujourd'hui

\section{Quand Sud Aviation se rapproche de Nanterre}

L'extrême gauche trotskyste argentine face à Mai 68

\section{Jean Baptiste Thomas}

\section{(2) OpenEdition}

\section{Journals}

Édition électronique

URL : https://journals.openedition.org/ideas/2296

DOI : 10.4000/ideas.2296

ISSN : 1950-5701

Éditeur

Institut des Amériques

Référence électronique

Jean Baptiste Thomas, «Quand Sud Aviation se rapproche de Nanterre », IdeAs [En ligne], 11 | 2018,

mis en ligne le 20 juin 2018, consulté le 20 octobre 2022. URL : http://journals.openedition.org/ideas/ 2296 ; DOI : https://doi.org/10.4000/ideas.2296

Ce document a été généré automatiquement le 20 octobre 2022.

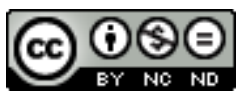

Creative Commons - Attribution - Pas d'Utilisation Commerciale - Pas de Modification 4.0 International - CC BY-NC-ND 4.0

https://creativecommons.org/licenses/by-nc-nd/4.0/ 


\title{
Quand Sud Aviation se rapproche de Nanterre
}

\author{
L'extrême gauche trotskyste argentine face à Mai 68
}

Jean Baptiste Thomas

11968 n'est pas seulement l'année de l'offensive du Têt menée contre les forces sudvietnamiennes et étasuniennes, et le mois de mai ne saurait simplement se caractériser comme le mois de la rébellion de la jeunesse estudiantine parisienne. Mai-juin 1968 représente le mouvement de grève le plus intense du mouvement ouvrier occidental du XX siècle et l'année 1968 est une sorte d'année «cardiaque » au niveau mondial au sens où elle coïncide avec le début d'une poussée ouvrière, populaire et de la jeunesse qui secoue l'Ouest, le Bloc de l'Est mais également les pays du Sud. Avec 1968, en quelque sorte, s'écroule la théorie selon laquelle il y aurait un découplage des temps de la révolution internationale, avec un centre capitaliste où le salariat, atone, aurait été intégré par le capitalisme, et où le seul espoir de transformation viendrait de la périphérie coloniale ou semi-coloniale.

2 C'est en ce sens que 1968 repose, au sein de la gauche radicale, plusieurs questions: celle du « sujet » ou des « acteurs » de la révolution (la paysannerie, les « damnés de la terre » ou les barbudos ou bien aussi, ou fondamentalement, le salariat, y compris dans les pays du Premier monde), celle du processus révolutionnaire et de son théâtre (rural et périphérique, ou urbain et ouvrier) ainsi que celle de "l'hypothèse stratégique " (guerre populaire prolongée ou grève générale insurrectionnelle) ${ }^{1}$.

Dans l'Argentine de 1968, alors sous la botte d'un régime militaire arrivé au pouvoir en 1966 et qui prétend avoir asséné un coup définitif au mouvement ouvrier et étudiant, il est frappant de constater, le peu d'écho, à première vue, du Mayo francés au sein des organisations politiques et syndicales de gauche. La presse commerciale relaie pourtant les informations venant de France. Plusieurs hebdomadaires, à commencer par Marcha, publié à Montevideo mais largement diffusé de l'autre côté du Río de la Plata, couvrent les événements et publient interviews de responsables politiques français et de dirigeants du Mouvement du 22 mars. Cependant, comme le souligne le philosophe argentin Nicolás Casullo, étudiant à Paris au moment des événements de mai, «à la 
différence de Cuba, du Vietnam ou de l'Algérie, "Paris 1968" n'a jamais été en Argentine (...) une pancarte, une affiche, une banderole ou un slogan étudiant, dans les quartiers populaires, dans le mouvement syndical, au sein des organisations [de gauche], qu'elles soient politico-militaires ou non, qu'elles adhèrent à un schéma insurrectionnaliste ou guérillériste $»^{2}$.

4 Si l'on se réfère en effet à un journal aussi largement diffusé au sein du mouvement étudiant et, surtout, de la gauche syndicale que CGT, il est assez symptomatique d'observer qu'alors que la revue couvre l'actualité internationale, il n'y est jamais fait mention de Mai 68, à une exception près. Il s'agit d'une allusion dépréciative et passablement populiste qu'y fait Roberto Grabois, dirigeant du Front étudiant national, organisation universitaire péroniste dont une bonne partie de la base, par la suite, rejoindra les organisations de surface de Montoneros. Pour Grabois, « ceux qui pensent que les travailleurs doivent appuyer la révolution des étudiants continueront à rêver de Paris, alors que l'histoire se passe [ici], à Avellaneda, à Tucumán, dans chaque quartier, dans chaque province de la patrie $»^{3}$.

Horacio Tarcus voit dans cette absence une sorte d'incompréhension de la base ouvrière péroniste face à un mouvement qui s'est élevé contre de Gaulle. À plusieurs reprises, une certaine tradition péroniste a, en effet, identifié l'homme du 18 juin 1940 à celui du 17 octobre 1943, date à laquelle Juan Domingo Perón, alors secrétaire au Travail du gouvernement Farrell et destitué sur pression des secteurs les plus conservateurs de l'establishment, est remis en selle par une grève générale et une vaste mobilisation populaire qui changent le visage de l'Argentine contemporaine. C'est ce dont attestent les slogans scandés par les cortèges syndicaux à l'époque de la visite du président français en Argentine, en octobre 1964, dans le cadre de sa grande tournée en Amérique latine. Le justicialisme est proscrit depuis le coup d'État de 1955 et les partisans de Perón profitent de la venue de De Gaulle en 1964 pour rendre hommage à leur général en manifestant au cri de « ¡De Gaulle-Perón, un solo corazón! » ou encore « ¡De Gaulle-Perón, Tercera posición! »4. Tarcus décèle également, dans ce manque d'intérêt relatif, la main des communistes. À l'époque, le Parti communiste argentin (PCA) s'empresse de traduire et de publier, dans la revue théorique du parti, Cuadernos de Cultura, les positions de Roger Garaudy condamnant l'extrémisme infantile et petitbourgeois du mouvement étudiant et des gauchistes français ${ }^{5}$.

6 Cela ne veut pas dire que de façon plus souterraine Mai 68 ne trouvera pas, au cours des mois suivants, un écho que l'on retrouve dans la multiplication de la traduction d'essais et d'ouvrages consacrés aux événements français publiés par plusieurs petites maisons d'édition proches de la nouvelle gauche ou de la revue cordobaise des gramsciens argentins Pasado y Presente, produite par des intellectuels en rupture avec le PCA, à l'image de José Aricó ou Juan Carlos Torre. Globalement, néanmoins, au moment des événements français, le «Mayo francés » est globalement passé sous silence ou analysé de façon très critique par le PCA ou la gauche péroniste.

7 C'est dans ce cadre qu'il est intéressant de souligner l'exception que représente la couverture des événements français par La Verdad et de El Combatiente. Les deux hebdomadaires, publiés clandestinement par le Parti Révolutionnaire des Travailleurs (également appelé PRT-La Verdad, PRT-LV) de Nahuel Moreno et par le Parti Révolutionnaire des Travailleurs (PRT-El Combatiente, du nom de son organe, PRT-EC), de Roberto Santucho, accordent au fil des pages de leurs éditions de mai et juin 1968 une importance décisive à la situation française, tant du point de vue du soulèvement 
étudiant que de la grève générale ${ }^{6}$. Les deux organisations, alors de taille réduite, mais qui seront appelées à jouer un rôle décisif au cours du cycle d'insubordination politique et social que connaît l'Argentine à partir de 1969 et que viendra clore brutalement le coup d'État de mars 1976, se sont séparées, quelques semaines auparavant, en mars 1968, autour de la question du lancement de la lutte armée. Le PRT-EC souhaite procéder à une mise en place rapide d'une guérilla rurale là où le PRT-LV défend une approche plus temporisatrice. Il n'en reste pas moins que les deux organisations sont liées au Secrétariat Unifié de la Quatrième Internationale (SUQI) dont les militants, en France, à l'instar d'Alain Krivine ou d'Henri Weber, interviennent au sein de la Jeunesse communiste révolutionnaire (JCR), fondée en 1966, et qui joue, dans les événements français de 1968, un rôle important, notamment dans le mouvement étudiant. Les deux hebdomadaires argentins reprennent et traduisent largement, dans leurs analyses, les rapports qui leur arrivent du SUQI ainsi que du Socialist Workers Party (SWP), sa section états-unienne.

Couverture de El único camino hasta el poder obrero y el socialismo, tiré du document du $\mathrm{IV}^{\circ}$ congrès du PRT de février 1968 et signé Carlos Ramírez (Mario Roberto Santucho), Sergio Domecg (Oscar Demetrio Prada) et Juan Candela (Félix Helio Prieto), slnd (edition clandestine) [1968]

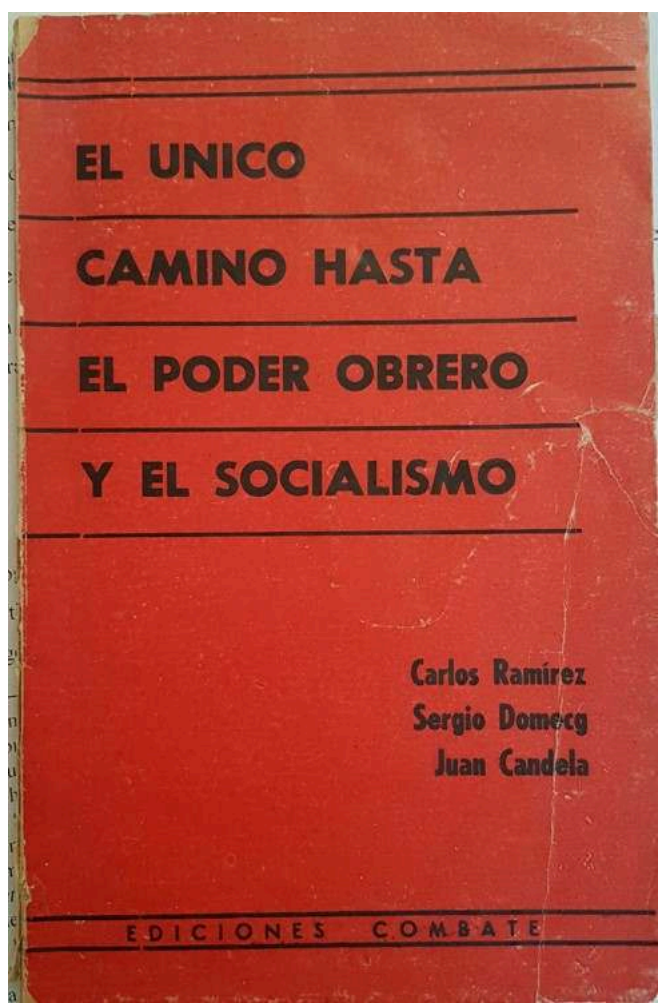

8 La distance géographique donne parfois lieu à des raccourcis assez cocasses. L'usine Sud Aviation de Bouguenais, près de Nantes, qui est à l'origine, avec Renault Cléon, de la poursuite de la grève après la journée du 13 mai, est ainsi située géographiquement, dans les colonnes de El Combatiente, près de Nanterre ${ }^{7}$. La confusion toponymique Nantes-Nanterre n'en reste pas moins politiquement assez juste, dans la mesure où $L a$ Verdad insiste sur l'alliance ouvrière-étudiante dont attestent les événements de Mai, avec le rôle détonateur de la jeunesse et la puissance, par la suite, de la grève générale ouvrière ${ }^{8}$. 
Il est assez intéressant, de ce point de vue, de souligner les conclusions stratégiques divergentes que tirent les deux PRT des événements qu'ils rapportent à partir des mêmes sources et que confirme, dans ses analyses à chaud, Roberto Santucho. Ce dernier est en effet présent à Paris, au cours des événements de mai, de retour d'un séjour de formation à Cuba, via Prague. Accompagné de militants de la JCR, il critique ce qu'il voit comme l'une des plus grandes limites du mouvement, à savoir l'absence de riposte armée de la jeunesse, sur les barricades, face aux forces de l'ordre gaulliste 9

Ainsi, plus que sur le processus en tant que tel, le PRT-EC insiste sur la violence de l'événement et de la période initiée par le «Mayo francés». Pour El Combatiente, Mai 68 n'est qu'un indicateur de plus à échelle internationale qui confirme combien la situation est mûre pour le lancement de la lutte armée. Ainsi, aux États-Unis, «la violence a liquidé la non-violence ${ }^{10}$ ", prônée par l'aile la plus modérée du mouvement des droits civiques. Au sein du mouvement de la jeunesse il est impossible d'arracher quoi que ce soit «si l'on ne dispose pas d'un moyen, si ce n'est à travers la confrontation brutale, violente (...) avec les forces de l'ordre $»^{11}$, souligne le PRT-EC en citant les propos d'un des dirigeants ouest-allemands de l'Union socialiste des étudiants (SDS). Cela vaut, bien entendu, également pour l'Argentine.

11 Le PRT-LV, en revanche, va commencer à réviser sérieusement les positions guérilléristes qu'il a pu défendre au cours des années 1960 sous l'influence de la Révolution cubaine et réitérées lors de son IV Congrès, à la veille des événements français.

Le mouvement étudiant français, soutient en effet La Verdad fin mai 1968, a démenti les spéculations et les théories [de ceux qui, à l'image] de Herbert Marcuse, soutiennent depuis des années que la classe ouvrière des pays avancés ne fera rien pour la révolution mondiale et que les intellectuels et les étudiants sont la nouvelle classe révolutionnaire. Ces honnêtes [intellectuels] révolutionnaires tiraient une conclusion hâtive d'un élément circonstanciel : la classe ouvrière était maintenue dans sa passivité par les appareils bureaucratiques et par le boom économique ${ }^{12}$.

Pour les morénistes, donc, Mai 68 marque le début d'une remontée du mouvement ouvrier à échelle internationale ainsi que d'une nouvelle séquence permanentiste. Les temporalités des processus révolutionnaires, longtemps désaccordées, entre un Sud en mouvement et un Nord relativement stable, entre 1948 et 1968, recommenceraient à battre au même rythme. Pour le PRT-LV, donc, « Mai 68, la rébellion de la jeunesse [à échelle internationale], la lutte sans quartier des masses vietnamiennes contre l'envahisseur yankee (...) font partie du contexte actuel qui caractérise l'Amérique latine $\aleph^{13}$. Par la suite, à l'encontre des positions majoritaires du SUQI, qui entend poursuivre l'orientation guérillériste pour l'Amérique latine, les morénistes appuient les positions du SWP états-unien qui soutient que Mai 68 annonce le retour « à la norme des révolutions prolétariennes classiques ${ }^{14}$, Moreno définissant comme " "normales" les révolutions qui ont pour centre de gravité le prolétariat industriel, les villes comme cadre géographique et l'insurrection urbaine comme axe de la lutte armée ${ }^{15}$, que ce soit dans les pays les plus développés ou dans les régions périphériques.

Ce débat sur la caractérisation de la situation internationale et ses répercussions, en Argentine, à la lumière de Mai 68, est particulièrement intense entre PRT-EC et PRT-LV. La discussion va d'ailleurs être relancée, entre les deux organisations et plus largement au sein de la nouvelle gauche argentine, quasiment un an jour pour jour après les événements français, par l'explosion du «Cordobazo ». 


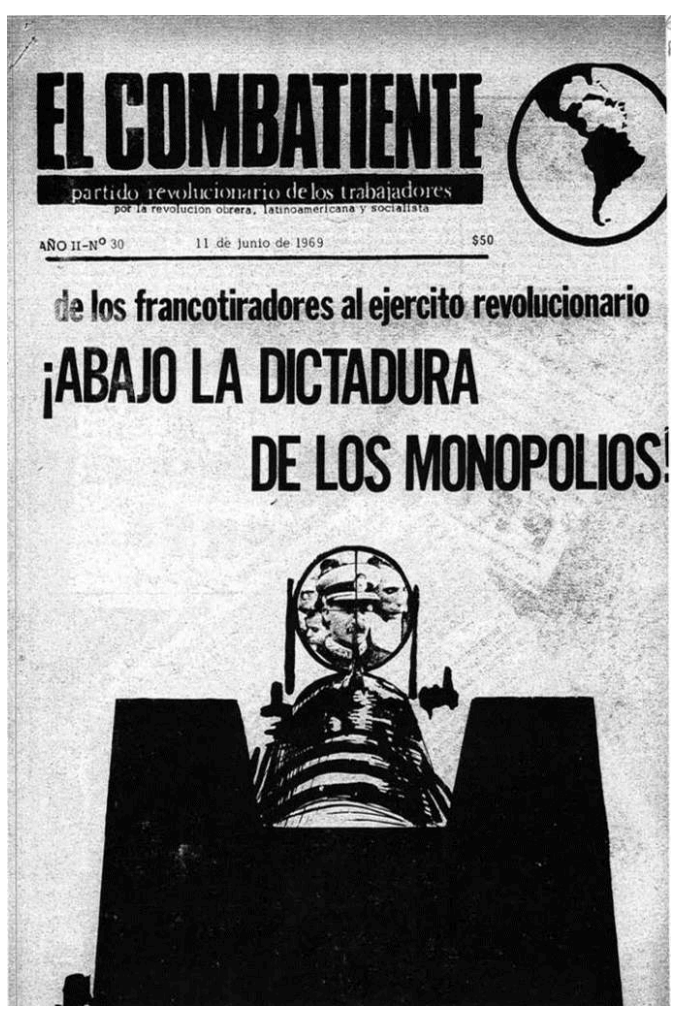

Le «Cordobazo » est une sorte de « Mai 68 » à retardement dont les protagonistes vont être le mouvement étudiant, les ouvriers de l'automobile et de l'énergie de la seconde ville du pays qui connait, fin mai 1969, une situation semi-insurrectionnelle. Celle-ci inaugure un processus de contestation ouvrière et étudiante qui va pousser la dictature argentine dans les cordes et forcer les militaires à opter pour une ouverture démocratique et permettre le retour du péronisme au pouvoir, seul courant à même de chevaucher le tigre de l'insubordination sociale et politique. Dans ce cadre, les événements de mai-juin 68 servent de première grille d'analyse à partir de laquelle la gauche trotskyste argentine va consolider ses choix politico-stratégiques, qui faisant le pari de la lutte armée, qui le pari de la construction d'une alternative politique à partir de la conflictualité croissante du monde du travail et de la jeunesse.

\section{BIBLIOGRAPHIE}

\section{Ouvrages}

Bensaïd, Daniel, « Stratégie et politique : de Marx à la III ${ }^{\mathrm{e}}$ Internationale » [2007], La politique comme art stratégique, Paris, Syllepse, 2011.

Casullo, Nicolás, París 68. Las escrituras, el recuerdo y el olvido, Buenos Aires, Manantial, 1998

Habel, Janette, conversation avec l'auteur, Paris, 18 mars 2018 
Tarcus, Horacio, «El Mayo argentino», Observatorio Social de América Latina n² 24, CLACSO, Buenos Aires, octubre 2008, p.161-180

Moreno, Nahuel, «Partido leninista o partido mandelista » [1973], El partido y la revolución, Buenos Aires, Antídoto, 1989

\section{Périodiques et bulletins internes}

Hansen, Joseph, « Assessment on the draft resolution on Latin America» [1969], International Information Bulletin. Discussion on Latin America (1968-1971), SWP, sl. nd. [1971], p. 23.

El Combatiente, édition clandestine, $\mathrm{n}^{\circ} 5 ; 9$ (1968)

International Viewpoint, New York, n² 20 (1968)

La Verdad, édition clandestine, $\mathrm{n}^{\circ} 136 ; 137 ; 250(1968 ; 1971)$

\section{NOTES}

1. Bensaïd, Daniel, «Stratégie et politique : de Marx à la III ${ }^{\mathrm{e}}$ Internationale » [2007], La politique comme art stratégique, Paris, Syllepse, 2011, p. 76-83.

2. Casullo, Nicolás, París 68. Las escrituras, el recuerdo y el olvido, Buenos Aires, Manantial, 1998, p. 46.

3. «Hablan los dirigentes estudiantiles», CGT n³3, Buenos Aires, 12/12/1968, p.3.

4. « De Gaulle-Perón, un même combat ! » et « De Gaulle-Perón, Troisième position ! ».

5. Tarcus, Horacio, «El Mayo argentino», Observatorio Social de América Latina ${ }^{\circ}$ 24, CLACSO, Buenos Aires, octubre 2008, p. 167-169.

6. Pour être tout à fait complet, il faudrait inclure le traitement des événements de mai et juin réalisé par Política Obrera, organe du petit groupe trotskyste du même nom (PO) lié, quant à lui, à l'Organisation Communiste Internationaliste (OCI) française. L'extension de cet article ne nous permet pas d'aborder ce cas.

7. Voir «¿Qué está ocurriendo en Francia?», El Combatiente n 9, éd. cland., 05/06/1968, p.2. L'erreur est due à la reproduction d'une erreur dans le texte original (What is happening in France »), écrit par Joseph Hansen, dirigeant du SWP américain, et publié dans International Viewpoint $n^{\circ} 20$, New York, 21/05/1968, p.458.

8. Voir notamment «La rebelión francesa conmueve el mundo", La Verdad $n^{\circ} 136$, ed. cland, 03/06/1968, p.1. Ainsi que « De la movilización estudiantil a la revolución socialista », La Verdad $\mathrm{n}^{\circ} 137$, ed. cland, 03/06/1968, p.6-7.

9. Habel, Janette, conversation avec l'auteur, Paris, 18 mars 2018.

10. «La violencia liquidó la no violencia», El Combatiente $n^{\circ}$ 5, éd. cland., 15/04/1968, p.1.

11. «Revolución juvenil», El Combatiente $n^{\circ}$ 8, éd. cland., 20/05/1968, p.7.

12. « De la movilización estudiantil a la revolución socialista », art. cit., p. 6.

13. «América Latina en la era de la revolución permanente», La Verdad n²50, éd. cland, 13/01/1971, p.6.

14. Hansen, Joseph, «Assessment on the draft resolution on Latin America» [1969], International Information Bulletin. Discussion on Latin America (1968-1971), SWP, sl. nd. [1971], p. 23.

15. Moreno, Nahuel, «Partido leninista o partido mandelista» [1973], El partido y la revolución, Buenos Aires, Antídoto, 1989. 


\section{AUTEUR}

\section{JEAN BAPTISTE THOMAS}

Jean-Baptiste Thomas est Maître de Conférences en histoire hispano-américaine contemporaine à l'Université de Poitiers (Département d'études hispaniques et hispano-américaines), membre du CRLA-Archivos (UMR 8132). Il travaille sur les questions d'insubordination ouvrière et sociale dans les années 1960 et 1970, dans le Cône Sud et depuis une perspective comparatiste. 